\title{
Comparison of Transmission Technologies with Regard to their Contribution to Power System Stability
}

\author{
S. Höhn ${ }^{1}$, A. Semerow ${ }^{1}$ and M. Luther ${ }^{1}$ \\ ${ }^{1}$ Chair of Electrical Energy Systems \\ University of Erlangen-Nuremberg \\ Konrad-Zuse-Straße 3-5, 91052 Erlangen (Germany) \\ E-mail: sebastian.hoehn@ees.fau.de
}

\begin{abstract}
The amount of power to be transmitted is increasing worldwide. Hence, a considerable number of bulk power transmission projects are going to be realized (e.g. [1]). Taking into account the recent technical development, three technologies are available for bulk power transmission: Direct current applications with voltage sourced or line commutated converters as well as the high-voltage AC technology up to $1200 \mathrm{kV}$. To select the most suitable technology for a particular project is often anything but trivial for the transmission system operator. Amongst other criteria, the influence on the electromechanical behavior of the power system is a decisive factor for network improvements that should be considered within the decision-making process.

This paper aims at categorizing and analyzing this rather abstract term as a major criterion. The goal is to provide an indication, which factors are needed to be evaluated in advance of the well-considered choice.

For that reason, several categories are introduced and thoroughly investigated within the scope of a theoretical approach. Where reasonable, simulation results are presented in order to enable the practical assessment of the impact on the electromechanical system behavior.

As a conclusion, it is recommended to perform concise ex-ante studies with regard to the electromechanical dynamics as a decision-making basis to identify the most suitable transmission technology for a particular project.
\end{abstract}

\section{Key words}

HVDC, HVAC, bulk power transmission, power system stability, electromechanical transients

\section{Introduction}

The introduction of electricity markets has led to an enormous enlargement of the power exchanges on transmission lines triggered by trading activities. Furthermore the contemporarily increasing penetration of renewable energy superimposes another effect: Conventional power plants are generally built close to large load centers, whereas the site of wind turbines, hydroelectric and solar power stations is defined by geographically allocated natural location factors [2]. These two developments have increased the demand for power transmission over long distances worldwide.

Currently, there are three different technologies for bulk power transmission: The three-phase high-voltage alternating current (HVAC) transmission system, the self-commutated high-voltage direct current transmission with voltage sourced converters (VSC-HVDC) and the classic line-commutated converter (LCC-HVDC) technology. In general, it is not obvious which of the transmission technologies is the most suitable for a specific bulk power transmission project. Therefore it is necessary to perform comprehensive studies in order to find out the most appropriate solution.

While most of the comparative approaches focus on economic issues (e.g. [3]), the influence of the transmission technology on the electromechanical system behavior has been also addressed as a crucial factor (e.g. [4], [5]). However, most of these approaches are rather vague. This paper seeks for establishing several criteria to comprehensively assess the electromechanical dynamic performance of the previously mentioned three transmission technologies, which could have enormous influences on the power system stability. An aphoristic theoretical analysis is performed on each of the introduced criteria.

Furthermore, this theoretical approach is accomplished by some additional basic simulations in order to determine the practical impact of a particular criterion on the electromechanical transients. For this purpose, elementary models are used to analyze the generic behavior in a simple test environment.

This publication overall includes a qualitative theoretical investigation on the dynamic performance of different transmission technologies together with practical simulations in a realistic environment to give a hint for the quantification of the theoretically stated influences of the transmission technologies on the electromechanical system behavior.

\section{General comparison of transmission technologies}

Since the first high-voltage three-phase transmission line was tested and commissioned in 1891, power systems worldwide primarily rely on the three-phase AC concept. Wherever this proven technology was stretched to its limits, DC concepts have been implemented for more than 60 years. In recent years the HVDC technology has succeeded in leaving its niche and competes with the 
conventional AC technology. In other words, the intersection, where both concepts are technically and economically feasible, is presently growing.

This leads to the necessity to evaluate which of the viable technologies is the best solution for a specific project. Being faced with complex problems, it makes sense to organize and categorize all involved matters. It's not sufficient to base the choice solely on the determination of the economical break-even distance (see [6]).

Keeping this in mind, that also other approaches already exist (e.g. [6)], Figure 1 shows the authors' attempt to give a structure to the comparison.

\section{Transmission technologies}

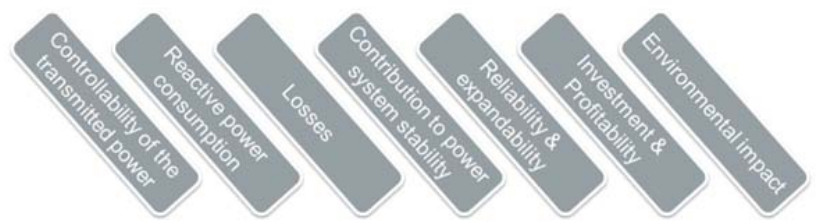

Figure 1: Criteria for a comparison of transmission technologies

All criteria that have direct influence on the investment or operational costs like losses or reactive power consumption are reckoned to be certainly the most important ones. However, factors that might seem to be less relevant can decide the success of a project. For example the environmental impact, i.e. noise, area required for lines and substations, etc., affect the public acceptance of system enhancements.

The criterion "contribution to power system stability" might be also a "hidden" decisive factor. It is obvious, that it is hardly possible to financially assess this category. However, it can bring crucial benefits or side effects. The measures can save investment costs as they could make other stabilizing measures redundant.

The effect of the transmission technology on the dynamic system behavior has often been addressed. The advantage of an HVDC connection for the power system's dynamic performance is emphasized in various publications. However, these statements are either used in general (e.g. [7]) or in order to promote a specific technology or project [4], [8]. Investigations illuminated by specific research results cannot be found. That is the motivation for creating a more tangible understanding of the complex and abstract criterion.

\section{Solutions for bulk power transmission}

Technical brochures that describe the transmission technologies are published by all relevant manufacturers for DC bulk power transmission solutions like ABB, Siemens, ALSTOM and General Electric. For a better understanding of the investigations it is recommendable to read the references given by the manufacturers. Nevertheless, some introductory information is given herein.

Transmission via HVAC concept is a proven technology. Lines with a nominal voltage of $750 \mathrm{kV}$ or $1000 \mathrm{kV}$ are being employed in large interconnected power systems. Due to their enormous reactive power consumption, depending on the load factor, it is out of the question, that these transmission lines need to be equipped with adequate compensation elements. These devices must be reasonably scaled and controlled by sophisticated control algorithms. FACTS based on power electronics are the favorable solution to provide these control functions.

The line commutated HVDC technology has been in operation for decades. The converters use thyristors whose firing impulses are adjusted by the converter controller. Both, rectifier and inverter require short circuit power for the commutation process. Due to the commutation and the delay of firing impulses, converters consume reactive power that must be provided by the grid or dedicated compensation elements. Filtering elements are necessary to reduce the distortion at the AC and the DC side.

In recent years, an alternative DC concept has made its way to the market: Voltage Sourced Converter (VSC) technology, which enables additional advantages in comparison to the classic concept. Its switchable IGBT semiconductors are capable of closing and interrupting the electrical circuit. Consequently, voltage angle and magnitude can be controlled almost independently. Reactive power support and active power are nearly decoupled. Furthermore a multi-level approach makes AC-filtering redundant or at least less expensive. Converter comprise of half bridge or full bridge power modules. The module type has influences on DC side short circuits as we can see later on. With having higher losses, VSC technology has only been rarely applied for bulk power transmission yet.

\section{Contribution to power system stability}

Several processes in modern power systems contribute to the increasing importance of power system stability: The penetration of renewable energies leads to temporary disconnection or permanent shutdown of conventional power plants. Hence, the power system might be operated with less inertia and reduced voltage and frequency control. Also power oscillation damping (POD) provided by conventional power plants might be reduced.

In addition, permanent system enlargements and the increasing power exchange due to market activities cause a significant approach towards the stability limits of interconnected power systems. In view of that fact, it is important to pay particular attention to maintaining power system stability.

For every modification of the power system the influence on the power system stability should be checked carefully. To integrate a bulk power transmission system is definitely such a noticeable modification. Even more, there is not only the chance to record the influences, but also to actively contribute to the power system stability.

A systematic approach helps to identify the possibilities a particular transmission technology provides and to assess the contribution to ensuring power system stability. Hence, a methodical structure with all important subjects is introduced in Figure 2. Each of the categories is explained and analyzed separately in the following chapters. 


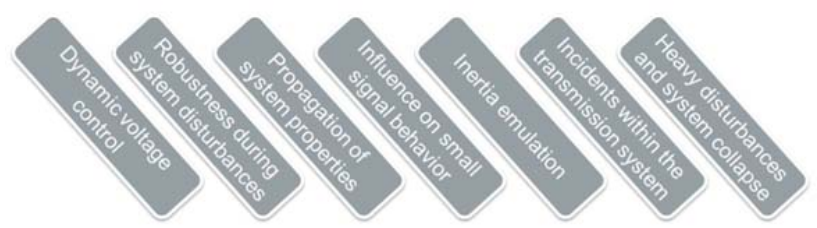

Figure 2: Subjects to assess the "contribution to power system stability" criterion for the comparison of transmission technologies

The theoretical approach is supplemented with some general simulations performed in an equivalent test scenario as shown in Figure 3. The load flow between the two grid equivalents A and B is carried out by six $380 \mathrm{kV}$ lines representing the conventional transmission system as well as by an overlay connection carrying $2 \mathrm{GW}$; the total amount of transmitted power is around $7.5 \mathrm{GW}$. The parameters for the bulk transmission in the model (e.g. geographical length, short circuit power, number of lines, etc.) are roughly representing the situation of a north to south transmission of electrical energy in Germany [1]. The behavior of the three transmission technologies is implemented by basic models. Neither the DC circuit nor the converter equations are included for the HVDC models. This allows a general assessment of the contribution to each criterion neglecting special and sophisticated features as well as control structures of specific transmission projects.

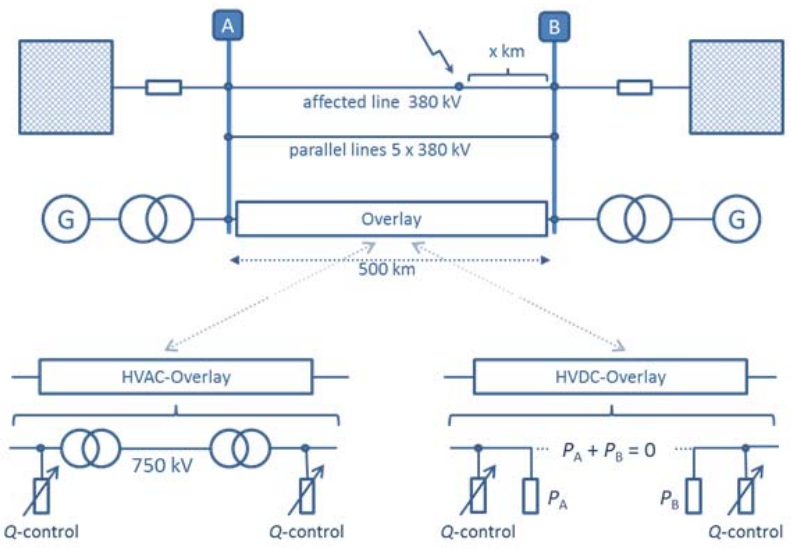

Figure 3: Scenario to assess dynamic performance

\section{A. Dynamic voltage control}

Voltage control is one of the main issues for the operation of power systems. A system is considered to be stable, if the quasi-stationary voltage remains within a defined voltage tolerance. However, incidents like shortcircuits cause temporary voltage drops. As the severity of the disturbance rises with the intensity of the voltage drop, countermeasures to support the voltage are required. This dynamic voltage control is an essential part of any common transmission code.

Generators, renewables, HVDC systems and FACTS controllers can provide the function of "reactive power boosting”. They inject reactive current to increase the voltage dip along the short circuit impedance during the fault. The magnitude of the additional reactive current is defined in the respective transmission code. (e.g. [11]) The voltage support is limited to the maximum admissible current of the reactive power boosting device.
Hence for the HVAC transmission line it is limited to the rated current of the compensating elements. For $750 \mathrm{kV}$ lines an approximation of $250 \mathrm{MVAr} / 100 \mathrm{~km}$ is given in [8]; a rough estimation for the compensation size of a LCC system is $Q_{\text {com }}=0.5 \cdot S_{\text {rated }}$ [9]. The LCC technology can give some additional support via adjustment of the firing angles. VSC can give the priority to reactive current that is only limited to the nominal current of the HVDC system.

The capability of the reactive power boosting depends on the ratio of rated power to short circuit power at the node the transmission system is connected to [10]. It is obvious, that VSC provides a superior support.

For the settings of the transmission model according to Figure 3, the reactive power support at rated voltage is 1 GVAr for LCC, 0.75 GVAr for HVAC and 2 GVAr for VSC at each terminal. Short circuit power of the grids A and $\mathrm{B}$ is $15 \mathrm{GVA}$ and $20 \mathrm{GVA}$, respectively. The contribution of the generators shall be negligible for the simulations.

Figure 4 shows the voltage dip at the busbar A and B caused by a three-phase short circuit $50 \mathrm{~km}$ to busbar B. Depicted are results for the three transmission technologies, as well as the simulation results without reactive power boosting. The dynamic voltage support is realized according to the German TransmissionCode 2007 [11]:

$$
\frac{i_{\text {additional reactive current }}}{i_{\text {rated }}}=2 \cdot \frac{\Delta U}{U_{\text {rated }}}
$$

It's obvious, that the impact of the voltage support is augmented by the amount of available reactive current.
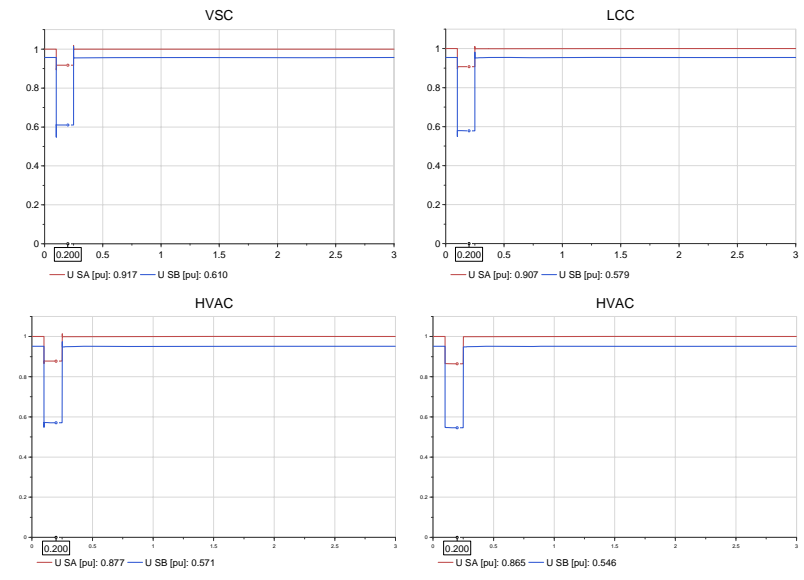

Figure 4: Impact of dynamic voltage support

As short circuit power is a criterion to select an adequate converter location, a high-short circuit power at busbar A and $\mathrm{B}$ is probable. However, as the ratio of apparent power of voltage supplying device to short circuit power is decisive for the impact of the reactive power boosting, the difference between the transmission technologies rises for weak connection points. For the simulation model, differences up to 0.2 pu were determined.

\section{B. Robustness during system disturbances}

The robustness of a transmission system is a valuable factor that can contribute to maintain the system stability during system disturbances.

The transfer function of an AC line between the nodes A and $\mathrm{B}$ is defined by the equation (2): 


$$
P=3 \frac{\left|\underline{U}_{\mathrm{B}(1)}\right|\left|\underline{U}_{\mathrm{A}(1)}\right|}{\left|\underline{Z}_{\text {line }}\right|} \cos \left(\varphi_{U_{\mathrm{B}}}-\varphi_{U_{\mathrm{A}}}+\varphi_{Z_{\text {line }}}\right)-\frac{\left|\underline{\mathrm{U}}_{\mathrm{B}(1)}\right|^{2}}{\left|\underline{Z}_{\text {line }}\right|} \cos \left(\varphi_{Z_{\text {line }}}\right) \text { (2) }
$$

The famous "nose" curve [12] as shown in Figure 5 can be derived from the transfer function and describes the voltage ratio $u=u^{U_{(1)}} / U_{\mathrm{A}(1)}$ as a function of transmitted active power $p$ and load factor $\varphi$.

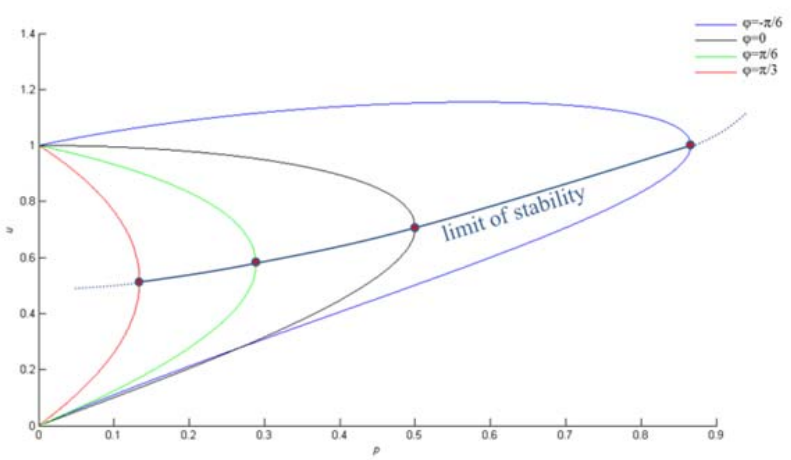

Figure 5: Nose curve for transmission stability

If the power demand at the receiving end of the line exceeds the limit of stability, the transmission line cannot satisfy the demand, the voltage declines rapidly and the voltage stability is jeopardized. Automatic line tripping is triggered when the voltage ratio falls below a security margin towards the limit of stability.

The LCC technology is a more robust technology as it cannot be overloaded. However, the commutation process depends on the voltage magnitude at the converters' AC voltages. Abrupt voltage drops can inhibit the extinction of a valve. Depending on the operation point a sudden voltage decline of $0.1 \mathrm{pu}$ at the inverter can cause a commutation failure. Capacitor commutated converters as part of the portfolio improve the robustness of the commutation process to 0.15 or 0.20 $\mathrm{pu}[4]$.

The VSC technology does not have such requirements for the operation. Even very low voltages can be handled without any restrictions. Especially during system disturbances it can provide valuable support for the interconnected system. It is not only possible to maintain the proper operability but also to actively provide voltage support or frequency control for applications with asynchronous or electrically weak coupled converter stations.

As they do not participate in cascading outages and due to their robust control concept, HVDC applications have proven their stabilizing effect at several severe disturbances, particularly in North America. However, the behavior at partial or even total system collapses must not be ignored. After power system separation an HVDC system that connects two temporarily asynchronous islands can adjust the transmitted active power in order to regain the load-generation balance of each island.

The use of light triggered thyristors minimizes the required energy for an unlimited black start capability [8]. Additionally, a VSC HVDC system provides the ability to energize a collapsed island as it requires neither reactive power nor short circuit power for the rectifier [4].

\section{Propagation of system properties}

Each transmission technology connects at least two nodes of the interconnected system. Hence, those nodes are coupled via the overlay connection and the regular power system. With the transmission technologies having different transfer functions for individual electrical properties, the coupling of the nodes depend on the transmission technology.

The transfer function for active and reactive power as properties of primary interest can be easily stated for each transmission technology:

For HVAC lines, the transmitted active power has been already mentioned. The transfer function for reactive power is given by:

$Q=3 \frac{\left|\underline{U}_{\mathrm{B}(1)}\right|\left|\underline{U}_{\mathrm{A}(1)}\right|}{\left|\underline{Z}_{\text {line }}\right|} \sin \left(\varphi_{U_{\mathrm{B}}}-\varphi_{U_{\mathrm{A}}}+\varphi_{Z_{\text {line }}}\right)-\frac{\left|\underline{U}_{\mathrm{B}(1)}\right|^{2}}{\left|\underline{Z}_{\text {line }}\right|} \sin \left(\varphi_{Z_{\text {line }}}\right)$

The voltages for LCC are coupled via

$U_{\mathrm{d} 1}=I_{\mathrm{d}} R_{\mathrm{d}}+L_{\mathrm{d}} \frac{\mathrm{d} I_{\mathrm{d}}}{\mathrm{d} t}+U_{\mathrm{d} 2}$,

the reactive power consumption of a converter is a function of the overlapping angle $u$ and the firing angle $\alpha$ :

$Q=\frac{3 \sqrt{2}}{\pi} U_{\text {rated }} \cdot I_{\mathrm{DC}} \frac{2 u+\sin 2 \alpha-\sin 2(\alpha+u)}{4(\cos \alpha-\cos (\alpha+u))}$

For VSC applications, the converters are almost decoupled by the DC capacitor. Voltage magnitude and angle can be controlled individually. However, there are operational and physical restrictions that inhibit arbitrary operation points. [9]

Converter equations and DC circuit equations connect rectifier and inverter mathematically. Therefore, a disturbance is always propagated from one side to the other. It's obvious, that the AC line has a considerably stronger coupling.

AC connections can also propagate other system properties like inertia or short circuit power. Assuming a high inertia at side B of the model (Figure 3) and an outage of generator $\mathrm{A}$, the transmitted inertia can counteract the frequency deviation as shown in Figure 6. The weaker the connection through the regular power system, the more stabilizing effect has the propagation of inertia.

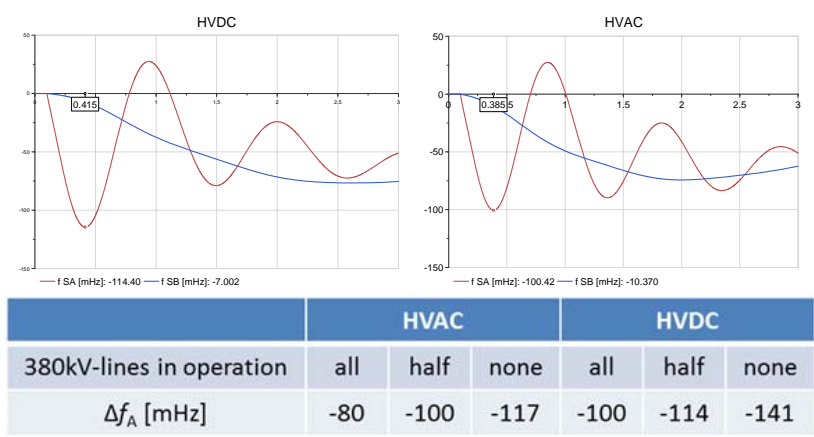

Figure 6: Impact of inertia propagation

The propagation of a short circuit voltage drop from side $\mathrm{B}$ to busbar A is summarized in Table 1. 
Table 1: Propagation of the voltage drop

\begin{tabular}{|c|c|c|c|c|c|c|}
\hline & \multicolumn{3}{|c|}{ HVAC } & \multicolumn{3}{c|}{ HVDC } \\
\hline 380kV-lines in operation & all & half & none & all & half & none \\
\hline$\Delta U_{\mathrm{A}}[\mathrm{pu}]$ & 0.20 & 0.14 & 0.10 & 0.13 & 0.07 & 0.03 \\
\hline
\end{tabular}

The results show that the AC overlay transfers electrical properties that can have harmful or beneficial impacts on the system stability. It depends on the scenario which of these effects is of higher importance.

\section{Influence on electromechanical oscillations}

Electrical power systems can be stimulated in their characteristic eigenfrequencies by small disturbances as well as by severe incidents like generator outages or short-circuits. Rising market activities and burgeoning penetration of renewable energies make the system prone to poorly damped power oscillations and therefore jeopardize the small signal stability of the system. Obviously, it calls for effective countermeasures.

Both, FACTS and HVDC devices, can contribute to a better oscillation damping by adjusting the firing and extinction impulses of the converter valves. The controller can be equipped with a power oscillation damping. LCC HVDC modifies the transmitted active power, whereas FACTS controllers use the reactive power to contribute to the damping. VSC systems can use both properties in order to counteract the oscillations. FACTS devices equipped with a battery storage system [12] can also use active power to damp oscillations.

Figures 7 and 8 demonstrate the basic concepts of a power oscillation damping (POD) employing reactive and active power control, respectively. The approach focuses on the $P(\delta)$ - diagram [12] for a generator according to equation (2). Assuming a sudden load loss, the electrical power is reduced abruptly as the angle of the stator voltage is forced to jump. As a consequence the rotor is accelerated until the electric power is equal to the mechanical power. The accelerating energy which is stored in the rotary motion is highlighted in red colors in the diagrams. The equilibrium is then retrieved by an oscillatory motion.

If the reactive power is injected during an accelerated motion, the voltage is higher than without. This increases the active power output and consequently reduces the accelerating energy (Figure 7).

After having detected an acceleration of a rotating machine, the active power support by a parallel feed-in reduces the drop of the stator voltage angle as shown in Figure 8 on the right side. Again, the accelerating energy (marked in red) is reduced. During the oscillatory motion to the point of equilibrium the active or reactive power should be in phase with the frequency deviation in order to achieve the maximum POD [5].

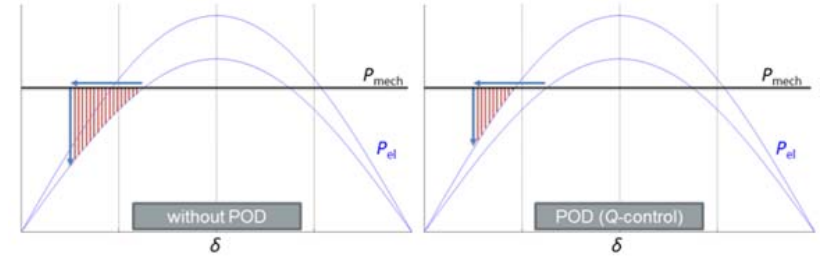

Figure 7: Effect of POD by reactive power control

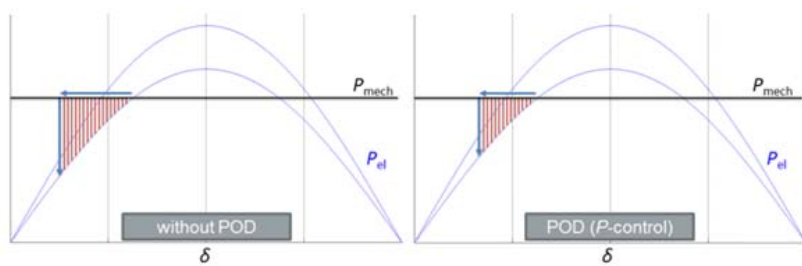

Figure 8: Effect of POD by active power control

The impact of the POD is not only a function of total amount of supplementary POD-power but also a question of the operating point of the machine at occurrence of the disturbance. It is evident that the reactive power control has more influence at higher rotor angles; at smaller ones, POD based on active power control is more promising.

Having either an AC or a DC connection also influences the eigenfrequencies of the power system. One inter-area mode exists for the model with generator A oscillating in phase opposition to generator $\mathrm{B}$. Table 2 shows the complex eigenvalues for the examined mode. As the $750 \mathrm{kV}$ line reduces the impedance between the antipodes, the oscillating frequency is smaller than for an HVDC overlay. However, the damping is worse for the HVAC overlay.

Table 2: Eigenvalues for the oscillatory mode in the model

\begin{tabular}{|c|c|c|c|c|}
\hline & \multicolumn{2}{|c|}{ HVAC } & \multicolumn{2}{c|}{ HVDC } \\
\hline 380kV-lines in op. & all & half & all & half \\
\hline eigenvalues & $-0.277 \pm j 3.637$ & $-0.428 \pm j 3.306$ & $-0.379 \pm j 3.258$ & $-0.599 \pm j 2.843$ \\
\hline
\end{tabular}

\section{E. Inertia emulation}

Synchronous generators contribute to power system stability by their inertial response to a frequency deviation. The torque equation for the rotary motion of a synchronous generator can be stated as follows:

$$
M_{\text {mech }}+M_{\text {el }}=M_{\text {damp }}+M_{\text {acc }}
$$

Neglecting the damping torque $M_{\text {damp }}$, it is evident that a change of the retarding electrical torque $M_{\mathrm{el}}$ disturbs the equilibrium and creates an accelerating torque $M_{\text {acc. The }}$. The mechanical torque $M_{\text {mech }}$ is a rather inert property that can be assumed to be constant for the first inertial response. The accelerating torque of a rotary motion is defined as $M_{\text {acc }}=J \cdot \frac{\mathrm{d} \omega}{\mathrm{d} t}$. The equation shows, that the inertia counteracts the change of angular velocity of the generator's rotor by use of the kinetic energy of its rotary motion.

Conventional power plants have been recently substituted by the high renewables. Consequently the system's inertia is getting reduced. New sources for artificial inertia can be generated. The prerequisite is an active power storage system connected by a controllable converter. Hence, wind turbines, photovoltaics and HVDC systems can provide this artificial inertia. Numerous technical studies have been performed in recent years. The focus lies on wind turbines (e.g. [14]) with the rotary speed as energy source; however also publications for inertia emulation with VSC HVDC are known $[16,17]$. Even the ENTSO-E draft Grid Code for HVDC [17] considers the "synthetic inertia": TSOs can require ancillary active power control functions.

The HVDC system must deliver a supplementary active power that corresponds to the accelerating power connected to $M_{\text {acc }}$. The additional energy can be provided 
by the electrostatic energy of the DC capacitances for VSC applications. In order to augment the available active power, it might be helpful to have a larger capacitance than required by normal operation [15]. While providing the same amount of additional active power the voltage declines less by considering this measure.

HVAC systems are only capable of transmitting, but not emulating inertia. However, there are approaches to equip FACTS devices with energy storage systems. [20] For LCC it's imaginable to use the DC reactor as energy storage for the artificial inertia.

\section{F. Incidents within the transmission system}

Most of the yet discussed criteria focus on the contribution of the transmission technology to power system stability. However, another crucial factor is the analysis, of how a fault that occurs on the overlay transmission line or converters affects the power system. Taking into account, that the converters comprise of a redundant number of power modules [4], [8], the most significant differences between the technologies are revealed by analyzing short-circuit occurrences on the transmission lines.

Table 3 gives an overview of the available fault clearing concepts for the transmission technologies. An AC distance protection device typically disconnects an affected line at $150 \mathrm{~ms}$. Auto-reclosing is usually initiated at 300-500 ms [18]. Different short circuit concepts exist for HVDC topologies: DC breakers can be applied to both LCC and VSC technologies. [19] Full bridge modules can inherently clear a short-circuit on the DC side. In addition they can restart the power transmission at a very low voltage enabling an earlier active power recovery. For a restart at nominal voltage it is stringently required that the insulating air is entirely deionized at the location of the short circuit.

Table 3: Fault clearing concepts for transmission systems [18-19]

\begin{tabular}{|l|c|c|c|c|}
\hline & \multicolumn{3}{|c|}{ HVDC } & HVAC \\
\hline clearing concept & AC-breaker & full bridge & DC-breaker & AC-breaker \\
\hline short circuit time & $30-60 \mathrm{~ms}$ & $<10 \mathrm{~ms}$ & $<10 \mathrm{~ms}$ & $150 \mathrm{~ms}$ \\
\hline interruption time & $1-2 \mathrm{~s}$ & $300-600 \mathrm{~ms}$ & $1-2 \mathrm{~s}$ & $300-500 \mathrm{~ms}$ \\
\hline
\end{tabular}

The short circuit is cleared by a breaker at the AC switchyard for HVDC systems without DC breaker or full bridge. As the converters are not connected to the grid during the outage of the DC line, they cannot be used to support the grid. With the converters connected they can be applied for stabilizing measures like dynamic voltage support, inertia emulation or POD in order to counteract the disturbance caused by the short-circuit or any other incident.

\section{Conclusion and outlook}

This treatise provides a structure to the abstract and often cited term of dynamic performance of the three available transmission technologies LCC, VSC and HVAC. Several criteria are introduced in order to assess the contribution of each concept to the power system stability. By this approach a thorough investigation on the influence on the dynamic system behavior is facilitated. As a specific transmission technology may bring benefits for the system stability, it is advisable to perform ex-ante studies assessing the influence on the electromechanical performance. A sufficient assessment can be made using elementary transmission technology and power system models. Even rudimentary imitation of particular controller functions can reveal valuable results. In the next step, investigations with regard to the proposed criteria should be performed for a specific project.

As the contribution to the power system stability is just one aspect amongst a number of categories for the evaluation of transmission technologies, it should be integrated in an overall comparison. However, it is difficult to financially assess the contribution to power system stability. Nevertheless dynamic system performance has to be regarded as a substantial cornerstone for the interoperability of interconnected power systems.

\section{References}

[1] ENTSO-E, “10-Year Network Development Plan 2012”, Brussels (2012)

[2] J. Schlabbach, K. Rofalski, "Power System Engineering”, WILEY-VCH, Weinheim (2008)

[3] Siemens AG, "High Voltage Direct Transmission - Proven Technology for Power Exchange, Erlangen” (2011)

[4] L. Carlsson, HVDC - A "firewall" against disturbances in high-voltage grids, ABB Review 3/2005, Ludvika (2005)

[5] P. Kundur, "Power System Stability and Control", 1st Edition, McGraw-Hill, New York (1994)

[6] D. Larruskain, I. Zamora et al, "Transmission and Distribution Networks: AC versus DC”, Marbella (2005)

[7] T. Halder, "Comparative Study of HVDC and HVAC for a Bulk Power Transmission”, Dindigul (2013)

[8] H. Huang, D. Retzmann et al, "UHV 1200 kV AC Transmission“, New Delhi (2007)

[9] M. Davies, M. Dommaschk et al, "HVDC PLUS - Basics and Principle of Operation“, Erlangen (2011)

[10] I. Erlich, F. Shewarega et al, "Effect of Wind Turbine Output Current during faults on Grid Voltage and the Transient Stability of Wind Parks“, Calgary (2009)

[11] VDN, "TransmissionCode 2007 - Network and System Rules of the German Transmission System Operators”, Berlin (2007)

[12] J. Machowski, J. Bialek, J. Bumby, "Power System Dynamics”, John Wiley \& Sons, Chichester (2008)

[13] E. Kimbark, "Direct Current Transmission”, John Wiley \& Sons, New York (1971)

[14] F. Gonzalez-Longatt, E. Chikuni et al, "Effects of the Synthetic Inertia from Wind Power on the Total System Inertia after a Frequency Disturbance”, Cape Town (2013)

[15] J. Zhu, C. Booth et al, "Inertia emulation control of VSCHVDC transmission system”, Beijing (2011)

[16] B. Silva, C. Moreira et al, "Provision of Inertial and Primary Frequency Cotnrol Services using Offshore Mulitterminal HVDC Networks”, London (2012)

[17] ENTSO-E, "ENTSO-E Draft Network Code on High Voltage Direct Current Connections and DC-connected Power Park Modules”, Brussels (2014)

[18] G. Ziegler, “Numerical Distance Protection”, Publicis Publishing, Erlangen (2011)

[19] M. Callavik, A. Blomberg et al, "The Hybrid HVDC Breaker”, Ludvika (2012)

[20] P. Ribeiro et al, "Energy Storage Systems for Advanced Power Applications”, Proceeding of the IEEE Vol.89, 2001 